\title{
Health-related quality of life in 4-to-6-year-old children with type 1 diabetes mellitus estimated by children and their mothers
}

\author{
Irina L. Nikitina ${ }^{1}$ (D) $\cdot$ Igor A. Kelmanson ${ }^{1}$ (D)
}

Received: 14 May 2021 / Revised: 29 July 2021 / Accepted: 10 August 2021 / Published online: 23 August 2021

(c) The Author(s), under exclusive licence to Springer-Verlag GmbH Germany, part of Springer Nature 2021

\begin{abstract}
Administration of pediatric Health Related Quality of Life (HRQoL) inventories frequently assesses both the child and parent perspectives in young children with type 1 diabetes mellitus (T1DM), but parent-proxy and child self-reports may differ, and little is known on these discrepancies. The aim is to evaluate HRQoL estimated by young children with T1DM and by their mothers, potential discrepancies in the children-maternal estimates and the factors influencing these discrepancies. Thirty-five 4-to-6-year-old children (19 boys) with T1DM admitted to the Pediatric Endocrinology Department were approached with the self-report KINDL questionnaire for children aged 4-6 years (Kiddy-KINDL for children). Their mothers were approached with the parental version (Kiddy-KINDL for parents). Both versions enable measuring child HRQoL in physical, emotional wellbeing, self-esteem, family, friends, everyday functioning, and the disease dimensions, as well as KINDL total on a 0-100 scale. Statistically significant differences were found between children's and maternal estimates on the KINDL total and "Disease" scales, in that the maternal proxy-reports produced lower values. A statistically significant difference between self- and proxy-reports was found for the KINDL "Emotional wellbeing" scale values, and the maternal proxy-reports yielded higher estimates compared with children's self-reports. These associations remained significant after adjustment for major potential confounders. Maternal education, maternal marital status, insulin regimen, and achievement of glycemic control modified the effect of child-maternal discrepancies.

Conclusion: Attempts should be made to improve parental understanding of child problems related to his/her disease with due account to individual family social and demographic characteristics.

\section{What is Known:}

- HRQoL in children with T1DM has been advocated as an important complementary outcome to clinical and laboratory markers.

- Self-and parental proxy-reports on HRQoL may differ, but little is known on these discrepancies and on the factors influencing them in young children with T1DM.

What is New:

- Mothers tend to underestimate general and disease-related components of HRQoL but likely to overestimate psychological wellbeing of their ill young children with T1DM.

- Maternal education, marital status, insulin regimen, and achievement of glycemic control modify estimations of HRQoL and child-maternal discrepancies.
\end{abstract}

Keywords Children $\cdot$ Diabetes mellitus $\cdot$ Health related quality of life $\cdot$ KINDL

Communicated by Peter de Winter

Igor A. Kelmanson

iakelmanson@hotmail.com

Irina L. Nikitina

nikitina0901@gmail.com

1 Department of Children's Diseases, Institute for Medical Education of the V.A.Almazov National Medical Research Centre, Akkuratova Str., 2, 197341 St. Petersburg, Russia

$\begin{array}{ll}\text { Abbreviations } \\ \text { ANOVA } & \text { Analysis of variance } \\ \text { BMI } & \text { Body mass index } \\ \text { HbA1c } & \text { Glycated hemoglobin } \\ \text { HRQoL } & \text { Health-related quality of life } \\ \text { T1DM } & \text { Type 1 diabetes mellitus }\end{array}$

Abbreviations

ANOVA Analysis of variance

BMI Body mass index

HbA1c Glycated hemoglobin

T1DM Type 1 diabetes mellitus 
Diabetes mellitus is a group of metabolic diseases characterized by chronic hyperglycemia resulting from defects in insulin secretion, insulin action, or both. Type 1 diabetes mellitus (T1DM) continues to be the main type of diabetes encountered in children and adolescents. Over $85 \%$ of all diabetes cases in individuals aged $<20$ years worldwide are T1DM [1]. T1DM is the most common childhood chronic illness [2], and, in some reports, its incidence is increasing most rapidly among children under 5 years of age [3]. Being diagnosed with T1DM permanently changes the life of children. Treatment has some requirements such as frequent insulin injections, daily blood glucose monitoring, diet plan, and regular physical activity. Also, acute and chronic complications related to diabetes may occur. Normative dependence upon parental caretaking in early childhood translates into considerable parental responsibility for the completion of daily diabetes management tasks [4]. All of these factors can adversely affect the health-related quality of life (HRQoL) in children and adolescents with T1DM [5-9].

Health-related quality of life (HRQoL) is an important construct assessing the impact of a medical condition [10]. It is a multidimensional concept including wellbeing in terms of patient's physical, emotional, mental, and social behaviors and is defined as the way the effects of a disease and/or its treatment are perceived by the patient [11]. It is agreed that enhancing quality of life and wellbeing is as important as metabolic control and prevention of secondary morbidity [12]. There is consensus on the importance of psychosocial support of children and adolescents to reach treatment recommendations, and HRQoL has been advocated as an important complementary outcome to clinical and laboratory markers $[13,14]$. Furthermore, a satisfactory HRQoL score is shown to be a marker of people's capacity and competence to manage their diabetes treatment and achieve treatment goals. Early detection of poor HRQoL scores is considered important, as it is hypothesized that these children are at risk of psychological maladjustment, reduced compliance and adherence to treatment, and poor metabolic control [15].

Wellbeing can be described in different forms by individuals, and the disease process may also be experienced differently. When evaluating quality of life, it should be considered that there are objective and subjective areas of HRQoL. Two people in the same situation objectively may have different perceptions of their HRQoL subjectively. Some researchers suggest that subjective assessment is more valuable because it reflects self-perception about the situation of individuals. Therefore, the evaluation of HRQoL perceived by parents as well as by the children is important to understand the children's HRQoL correctly. Parents of young children may experience anxiety and distress related to coping with the particular diabetes responsibilities for this age group [16]. The pediatric transactional theory posits reciprocal interactions between children's health and behavior and parents' characteristics and experiences [17]. Therefore, administration of pediatric HRQoL inventories frequently assesses both the child and parent perspectives (proxy) of the child's HRQoL. However, parent-proxy and child self-reports often differ [18, 19]. Little is known about what discrepant scores mean and what they may indicate in relation to child health outcomes, especially in young children. This "proxy problem" has been debated in the HRQoL literature $[18 ; 20]$, but no conclusion has been reached on how to handle the apparent discrepancies. Further, analysis of a variety of demographic and disease-related factors such as age, gender, and education varied in terms of how they associated with the direction of discrepancies across the multiple studies, when reported. The direction and magnitude of HRQoL discrepancies remain unclear, and there are no data on the potential importance of discrepant scores within families in relation to clinical outcomes.

To address the issue and bridge the gap, this study aimed at evaluation of HRQoL estimated by young children with T1DM and their mothers and at evaluation of potential discrepancies in the children-maternal estimates and the factors influencing these discrepancies.

\section{Patients and methods}

\section{Participants}

Participants were recruited from children aged 4 to 6 years with diagnosed T1DM who were under supervision at the Pediatric Endocrinology Department of the V.A.Almazov National Medical Research Centre, St. Petersburg, and who were scheduled for admission to the Department in 2020 for routine check-up and therapy correction. Inclusion criteria were that all children had been diagnosed with T1DM at least 1 year previously to their planned admission, and they represented urban environment. Children with coexisting inborn malformations, chronic diseases other than T1DM, and psychiatric diseases and those with coexisting acute disease during the study were excluded. Ninety-four children met the inclusion criteria. For ethical considerations, we limited the sample size to reasonable number of participants in order to reach a balance between the study's value and the burdens accepted by its participants. Required sample size computation was performed using G*Power 3.1.5 statistical software [21]. The study was targeted at determining a medium standardized effect size for the differences between two means (Cohen's $d=0.5$ ), based on the two-tailed test aimed at finding difference between two dependent means (matched pairs), with the $\alpha$ error equal to 0.05 , and the power $(1-\beta)$ equal to 0.8 . This yielded the desired sample size equal to 34 children. Ninety-four eligible candidates 
for selection were numbered in sequence in advance, and 40 children were selected from the list as a simple random sample without replacement using WinPepi 3.26 program [22]. On admission, these 40 selected children and their parents were approached with a request to participate in the scientific study. Of the 40 selected, 35 (88\%) agreed to take part in the study, and they constituted a study group. Patients from the families refusing participation did not differ from the participants in terms of age, gender, socioeconomic status, duration of diabetes, and glycemic control.

\section{Measures}

\section{Clinical and socio-demographic characteristics}

Clinical findings were extracted from the patients' medical records. The mothers were asked to complete the questionnaires addressing child, maternal, demographic major characteristics. To ensure reliability and to minimize recall bias, attention was paid to the thorough analysis of pre-existing medical records. Demographic variables included child sex, age, birth weight, weight and body mass index (BMI) at study, and number of siblings. Maternal marital status was classified as married, cohabiting, divorces, widowed, or single. Information on parental employment was collected. The maternal educational level was defined as the highest level of completed education and categorized as basic education (elementary school), secondary education (secondary school, college), higher education (institute, academy or university), or incomplete higher education. Clinical variables included duration of diabetes at the time of study, insulin regimen, and glycemic control. Insulin regimen was categorized as insulin pump or basal-bolus administration.

\section{Laboratory findings}

Blood glucose and HbA1c levels were measured at admission as a part of the routine laboratory investigation in T1DM children. HbA1c was analyzed using Bio-Rad D-10 analyzer. According to current clinical practice consensus guidelines, $\mathrm{HbA} 1 \mathrm{c}$ values below $7 \%$ were defined as good metabolic control. This target value has been chosen with the aim of avoiding long-term microvascular and macrovascular complications of diabetes while also avoiding severe hypoglycemia and the adverse central nervous system changes associated with both hypoglycemia and hyperglycemia [23].

\section{Questionnaire for evaluation of $\mathrm{HRQ} \mathrm{oL}$}

The KINDL questionnaires were used to evaluate HRQoL [24]. The KINDL questionnaire satisfies the demand for taking into account progress during child development and the principle of patient-generated data collection by providing different versions of the questionnaire for different age groups and both a self-report version and a proxy version. The common practice of modifying a measure originally designed for adults to make it suitable for children was avoided in this inventory. The original German KINDL questionnaire was duly validated and translated into several languages, including Russian. The psychometric testing of the KINDL indicate adequate to good reliability and convergent and discriminant validity of this inventory [24], in particular among pre-school children [25, 26], and the KINDL was proved efficient in the studies on HRQoL in children with T1DM [12]. Russian versions of the self-report KINDL questionnaire for children aged 4-6 years (Kiddy-KINDL for children) and for parents of children aged 3-6 years (KiddyKINDL for parents) were retrieved (https://www.kindl.org/ english/language-versions/russian/) and used in this study.

The self-report version of the Kiddy-KINDL questionnaire consists of twelve Likert-scaled items, two for each of six dimensions: physical wellbeing, emotional wellbeing, self-esteem, family, friends, and everyday functioning (school or nursery school/kindergarten). The response categories of the Kiddy-KINDL cover 3 levels $(1=$ never, $2=$ sometimes, $3=$ very often , and the children are to be questioned in a face-to-face interview. The sub-scales of these six dimensions can be combined to produce a total score.

The parents' version of the Kiddy-KINDL consists of 24 items, four for each of the six above indicated dimensions, and the response categories cover 5 levels $(1=$ never, $2=$ rarely, $3=$ sometimes, $4=$ often, $5=$ all the time). In order to make up for the potentially lower information content of the self-reported responses by young children, the parents' version of the Kiddy-KINDL contains a further 22 items which can be treated as a sub-scale in their own right.

An additional sub-scale entitled "Disease" aimed at completion in case of prolonged illness or hospitalization, as in this present study, is included both into self-report and parents' forms and consists of six items that measure the child's quality of life with respect to his or her illness. Similar to other sub-scales, the response categories cover 3 levels in the self-report version, while they cover 5 levels in the parent's version.

The scores achieved on the individual KINDL sub-scales and the KINDL total score represent a quantification of the subject's health-related quality of life from the respondent's point of view. Higher sum score values are indicative of better HRQoL.

For each item, the children were asked to mark the response that came closest to their own personal experiences. Mothers of the children were asked to complete the KINDL questionnaire as proxies, i.e., to judge the children's quality of life from their own point of view. 
The KINDL questionnaires were analyzed by adding the item responses marked on each sub-scale. Certain KINDL items are worded in such a way that a higher item score implies a poorer health-related quality of life. Reversing the values of these items was necessary in order to ensure that higher scores correspond to a higher HRQoL for all the KINDL items and sub-scales [27].

To ensure compatibility between self-report and parents' report, scores were summarized and transformed to a 0-100 scale using the following suggested formula [27]:

[(Sub-scale score - lowest possible score)/possible range of raw score $] \times 100$.

\section{Statistical analysis}

Descriptive and analytical statistics were used in data analysis. The internal consistency of both tools (selfreport and parent's KINDL versions) was assessed by Cronbach's $\alpha$ and McDonald's $\omega$ coefficients. To avoid dependence on normality of distributions, the Wilcoxon non-parametric matched-pairs signed-rank tests were run to measure differences in the KINDL sub-scales and the KINDL total score according to self- and proxy-reports. The rank-biserial correlation $\left(r_{B}\right)$ was considered as a measure of an effect size, coefficients equal to 0.1,0.3, and 0.5 referring to small, medium, and large effect sizes, respectively [28]. To account for multiple comparisons of the KINDL sub-scales, false discovery rate was computed for each comparison ( $q$ value or adjusted $p$ ) using the Benjamini-Hochberg correction [29]. The mixeddesign ANOVA (repeated-measures with a betweenfactor ANOVA) was further used to test for significant differences between paired measures adjusted for possible confounding effect(s) of several clinical and demographic characteristics and to explore possible interactions, in that the two paired measurements (either self- or proxy-report) were entered into the model as the repeated measurements, while the confounding/modifying factor in consideration was included into the model as either a between-subject factor (for a category variable) or as a covariate (for a continuous variable) [30]. Partial $\eta^{2}$ was used a measure of the effect size with the values equal to $0.01,0.06$, and 0.14 referring to small, medium, and large effect sizes, respectively [28]. The models were checked for potential violation of homogeneity of variance assumption using Levene's test. When an effect of a between-subject factor was found significant, post hoc pairwise comparisons with Tukey correction were performed.

The value for significance was set at $p<0.05$. Statistical analyses were performed using the JAMOVI statistical software version 1.6 [31].

\section{Results}

Table 1 is the summary of the major clinical and demographic characteristics of the patients and families. The study comprised 16 girls and 19 boys with disease duration ranging between 1 and 5 years (mean 2.1). Twenty-two patients received basal-bolus insulin, while 13 were on insulin pump. The HbAlc level in the patients ranged between 5.5 and $11.2 \%$ (mean 7.4); in 19 children, it was below 7\%, which was considered as an achieved glycemic control.

Children and their mothers did not have any problems in understanding the language, the meaning of the questions in the KINDL questionnaires, and in selecting appropriate answers. The values of internal consistencies of the

Table 1 Patients' major characteristics. Absolute figures (\%) or median (interquartile range)

\begin{tabular}{|c|c|}
\hline & Overall $(N=35)$ \\
\hline \multicolumn{2}{|l|}{ Sex } \\
\hline Girls & $16(46 \%)$ \\
\hline Boys & $19(54 \%)$ \\
\hline Age, years & $5.0(1.0)$ \\
\hline Birth weight, $g$ & $3118(321)$ \\
\hline Weight at study, kg & $21(4.5)$ \\
\hline Height at study, $\mathrm{cm}$ & $115(10.3)$ \\
\hline BMI, $\mathrm{kg} / \mathrm{m}^{2}$ & $16.6(2.9)$ \\
\hline BMI z-score* & $1.3(0.7)$ \\
\hline T1DM duration, years & $2.0(1.5)$ \\
\hline $\mathrm{HbA} 1 \mathrm{c}, \%$ & $6.9(2.5)$ \\
\hline \multicolumn{2}{|l|}{ Glycemic control (HbA1c <7\%) } \\
\hline Yes & $19(54 \%)$ \\
\hline No & $16(46 \%)$ \\
\hline \multicolumn{2}{|l|}{ Insulin regimen } \\
\hline Basis-bolus & $22(63 \%)$ \\
\hline Insulin pump & $13(37 \%)$ \\
\hline \multicolumn{2}{|l|}{ Siblings } \\
\hline 0 & $20(57 \%)$ \\
\hline 1 & $8(23 \%)$ \\
\hline 2 & $5(14 \%)$ \\
\hline 3 & $2(6 \%)$ \\
\hline \multicolumn{2}{|l|}{ Marital status } \\
\hline Married & $22(63 \%)$ \\
\hline Divorced & $7(20 \%)$ \\
\hline Widow & $1(3 \%)$ \\
\hline Cohabiting & $5(14 \%)$ \\
\hline \multicolumn{2}{|l|}{ Maternal education } \\
\hline Higher & $21(60 \%)$ \\
\hline Incomplete higher & $11(31 \%)$ \\
\hline Secondary & $3(9 \%)$ \\
\hline Maternal age at study, years & $27.0(5.0)$ \\
\hline
\end{tabular}

*Values are based on WHO standards (birth to 60 months) and WHO reference 2007 (61 months to 19 years) 
Table 2 Self- and proxy-reports on total KINDL questionnaire and KINDL scales. Median (interquartile range)

\begin{tabular}{lccccc}
\hline KINDL scale & Self-report & Proxy-report & $P$ (Wilcoxon W) & $q$ & $\begin{array}{l}\text { Effect size (rank } \\
\text { biserial correlation) }\end{array}$ \\
\hline The KINDL total score & $69.4(27.8)$ & $42.1(20.8)$ & $\mathbf{< . 0 0 1}$ & $\mathbf{0 . 0 0 7}$ & .886 \\
Physical functioning & $75.0(37.5)$ & $56.3(12.5)$ & $\mathbf{. 0 3 1}$ & 0.055 & .424 \\
Emotional wellbeing & $75.0(62.5)$ & $87.5(31.3)$ & $\mathbf{. 0 0 7}$ & $\mathbf{0 . 0 2 5}$ & .540 \\
Self-esteem & $69.0(25.0)$ & $62.2(12.3)$ & .072 & 0.102 & .349 \\
Family & $75.0(50.0)$ & $81.3(15.6)$ & .112 & 0.132 & .333 \\
Friends & $78.0(37.5)$ & $81.3(21.9)$ & .558 & 0.564 & .126 \\
Everyday functioning & $75.0(50.0)$ & $62.5(18.8)$ & .788 & 0.696 & .058 \\
Disease & $53.2(37.5)$ & $41.7(33.3)$ & $\mathbf{. 0 1 9}$ & $\mathbf{0 . 0 4 5}$ & .454 \\
\hline
\end{tabular}

Statistically significant differences marked in bold self-report KINDL and parent's KINDL questionnaires and their specific sub-scales were acceptable-to-good ranging between 0.695 and 0.953 .

Table 2 shows the distributions of self- and proxy-reports on total KINDL questionnaire and different KINDL scales. Overall, a statistically significant difference was found in total quality of life estimates between a child and his/ her mother, in that the maternal proxy-reports produced lower values. Likewise, compared with children, the mothers reported statistically significantly lower values on the KINDL Disease scale. Large effect sizes were found for these differences. A statistically significant difference between self- and proxy-reports was found for the KINDL "Emotional wellbeing" scale values with large effect size; however, unlike the KINDL total and the KINDL Disease scores, the maternal proxy-reports on the KINDL Emotional wellbeing score yielded higher estimates compared with children's self-reports. A statistically significant difference between self- and proxy-reports was also found for the KINDL "Physical functioning" scale values, but this difference failed to retain its significance after correction for multiple comparisons.

The mixed-design ANOVA was further performed to test for significant differences between paired measures on total KINDL scores, the Disease and the Emotional wellbeing scores adjusted for possible confounding or modifying effect(s) of several clinical and demographic characteristics, including child sex, T1DM duration, siblings in a family, maternal education, maternal marital status, insulin regiment, and glycemic control. The two paired measurements (self- and proxy-reports) were entered into the model as the repeated measurements, while the confounding/modifying factor in consideration was included into the model as a between-subject factor (if it was a categorical) or as a covariate (if it was a continuous).

No statistically significant effects on the differences between the paired total KINDL measurements and the paired KINDL Disease measurements were found for child sex, T1DM duration, siblings in the family, and maternal age at study. Statistically significant within-subject effects related to the source of information (either self- or proxyreport) remained significant after adjustments for the above between-subject variables. A statistically significant effect on the total KINDL measurements was found for the maternal marital status as a between-subjects variable but was not found for the paired KINDL Disease measurements (Supplementary Table 3). The two paired total KINDL measurements (either self- or proxy-reports) retained its significance as a source of within-subject variance after marital status was included into the model. There was also a statistically significant interaction between the sources of total KINDL reports and maternal marital status (Fig. 2A). Following up this interaction indicates that the children and their mothers provided higher estimates on the total KINDL score when the mothers were married. However, the contrast between the self- and the proxy-estimates was more prominent when the mothers were married. Post hoc pairwise comparisons found that the values of the KINDL total scores were the highest in the self-reports of the children of the married mothers, and these values were statistically significantly higher than the values in the children whose mothers were not married (mean difference $=19.02, t(45.9)=3.21$, $p_{\text {tukey }}=0.012$ ). Statistically significant between-subjects effect was also found for maternal education (either higher or not) (Supplementary Table 3). The source of information on the KINDL total score (either self- or proxy-report) as a within-subjects effect retained its significance after adjustment for maternal education. There was also a statistically significant interaction between the source of report and maternal education (Fig. 2B). Following up this interaction indicates that the maternal estimates of the KINDL total score were always lower than those of their children, more so for those in maternal higher education. In the cases of maternal higher education, both children and their mothers reported lower values on the KINDL total score. Post hoc pairwise comparisons found that the children of the mothers 
with higher education had statistically significantly lower values on the KINDL total score than the children whose mothers had educational level other than higher (mean difference $\left.=-19.84, t(46.1)=-3.36, p_{\text {tukey }}=0.008\right)$. A statistically significant between-subjects effect was found for the insulin regimen (insulin pump vs. routine base-bolus administration) (Supplementary Table 3). The source of information on the KINDL total score (either self- or proxy-report) as a within-subjects effect retained its significance after adjustment for the insulin regimen. There was no statistically significant interaction between the paired differences in child-maternal reports and the insulin regimen. Therefore, the contrasts between the self- and the proxy-reports were similar under any insulin regimen, but the children receiving insulin via insulin pump and their mothers reported higher values on the KINDL total score than the pairs when the children were on a routine insulin administration. Post hoc pairwise comparisons found that the children on insulin pump had statistically significantly higher values on the KINDL total score than the children on a routine insulin regimen $\left(\right.$ Mean difference $\left.=18.12, t(47.0)=3.01, p_{\text {tukey }}=0.021\right)$. Similarly, glycemic control was found to have a significant between-subjects effect, and paired within-subject differences retained significance after adjustment for glycemic control (Supplementary Table 3). The level of HbA1c was found to have a statistically significant effect when it was included into the model as a continuous covariate rather than a dichotomized "glycemic control" variable $(p<0.001)$. Statistically significant interaction was found between glycemic control and the source of information. In the cases of accomplished glycemic control, both children and their mothers reported higher values on the total KINDL score. Post hoc pairwise comparisons found that the children who have achieved glycemic control reported statistically significantly higher estimates on the KINDL total score than the children in whom this control has not been accomplished $\left(\right.$ mean difference $\left.=26.74, t(49.2)=5.13, p_{\text {tukey }}<0.001\right)$; likewise, the mothers of those children who have reached glycemic control reported significantly higher estimates than the mothers of the children without glycemic control (mean difference $=15.16, t(49.2)=2.91, p_{\text {tukey }}=0.027($ Figs. 1D and 2).

Statistically significant between-subjects effect was found for the maternal education (either higher or not), and the source of information on the KINDL Disease score (either self- or proxy-report) as a within-subjects effect retained its significance after adjustment for maternal education (Supplementary Table 3). No statistically significant interaction between the source of information and maternal education was found. Both the children of the mothers with higher education and their mothers reported lower values on the KINDL Disease score than those pairs where the mothers did not have higher education (Fig. 2A). A statistically significant between-subjects effect was also found for the insulin regimen (insulin pump vs. routine administration). The source of information on the KINDL Disease score (either self- or proxy-report) as a within-subjects effect retained its significance after adjustment for the insulin regimen. There was a statistically significant interaction between the paired differences in child-maternal reports on the Disease score and the insulin regimen (Supplementary Table 3). Following up this interaction indicates that the estimates were higher
Fig. 1 Relationships between certain modifying factors, self- and proxy-estimates on the KINDL total score. Estimated marginal mean values and $95 \%$ confidence intervals
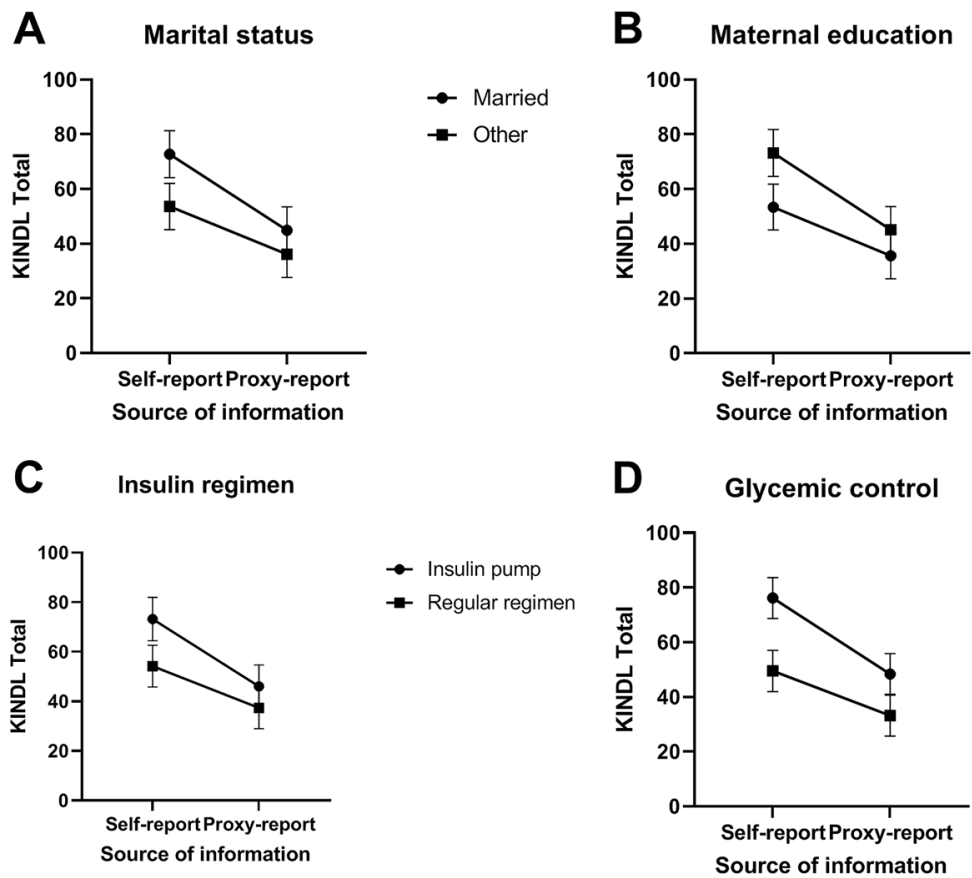

D Glycemic control

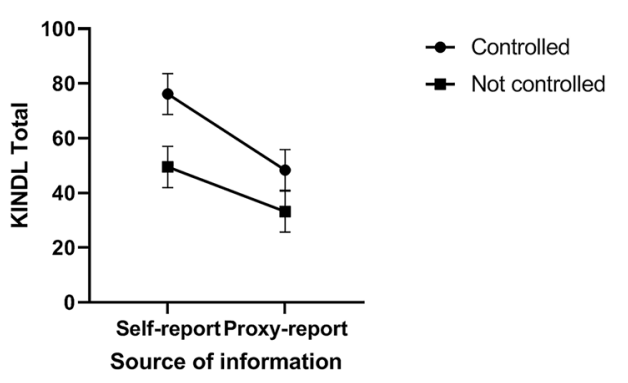



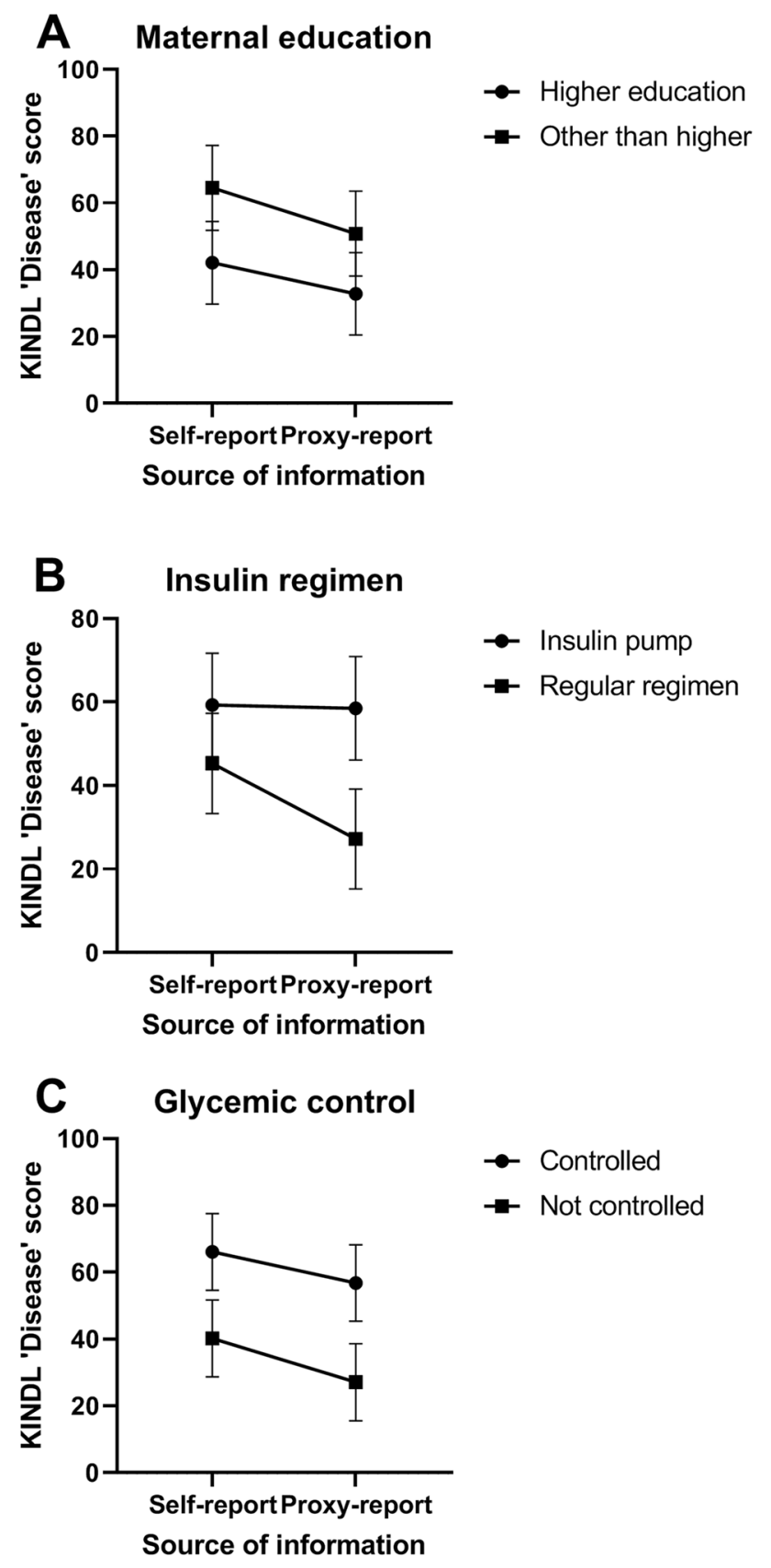

Fig. 2 Relationships between certain modifying factors, self- and proxy-estimates on the KINDL "Disease" score. Estimated marginal mean values and $95 \%$ confidence intervals

both in the children who were on a pump regimen and in their mothers, and the contrast between the estimates in these pairs was insignificant. The estimates in children on a routine insulin regimen and in their mothers were lower, and in these pairs, the contrast between the self- and the proxy-estimates was more remarkable and significant. The mothers of children on a routine insulin regimen provided statistically significantly lower estimates than the mothers whose children were on a pump, mean difference $=31.2$, $t(45.7)=3.651, p_{\text {tukey }}=0.004$ (Fig. 2B). Glycemic control was found to have a significant between-subjects effect, and paired within-subject differences retained their significance after adjustment for glycemic control. The level of HbA1c was found to have a statistically significant effect when it was included into the model as a continuous covariate rather than a dichotomized glycemic control variable $(p<0.001)$. There was no statistically significant interaction between glycemic control and the source of information on the KINDL Disease score. In the cases of accomplished glycemic control, both children and their mothers reported higher values on the KINDL Disease scores than in the cases where glycemic control was not achieved. Maternal estimates were lower than children's self-reports both in cases of accomplished and non-accomplished glycemic control (Fig. 2C).

No statistically significant between-subject effects were found when the paired KINDL Emotional wellbeing measurements were considered, and the following variables were explored for the between-subjects effect: child sex, T1DM duration, siblings in a family, and maternal age at study. As well, no statistically significant effect was found for the maternal marital status and insulin regimen. Statistically significant between-subjects effect was found for maternal education (either higher or not) (Supplementary Table 3). The source of information on the KINDL Emotional wellbeing score (either self- or proxy-report) as a within-subjects effect retained its significance after adjustment for maternal education. There was also a statistically significant interaction between the source of report and maternal education (Fig. 3A). Following up this interaction indicates that the maternal estimates of the KINDL Emotional wellbeing score were always higher than those of their children, more so for those in maternal higher education. In the cases of maternal higher education, both children and their mothers reported lower values on the KINDL Emotional wellbeing score. Post hoc pairwise comparisons found that the children of the mothers with higher education had statistically significantly lower values on the KINDL Emotional wellbeing score than the children whose mothers had educational level other than higher (mean difference $=-32.92, t(57.8)=-4.402$, $\left.p_{\text {tukey }}<0.001\right)$. Glycemic control was also found to have a significant between-subjects effect, and paired within-subject differences retained their significance after adjustment for glycemic control. The level of HbAlc was found to have a statistically significant effect when it was included into the model as a continuous covariate rather than a dichotomized glycemic control variable $(p<0.001)$. There was no statistically significant interaction between glycemic control and the source of information on the KINDL Emotional wellbeing score. In the cases of accomplished glycemic control, both children and their mothers reported higher values on the KINDL Emotional wellbeing scores than in the cases where 

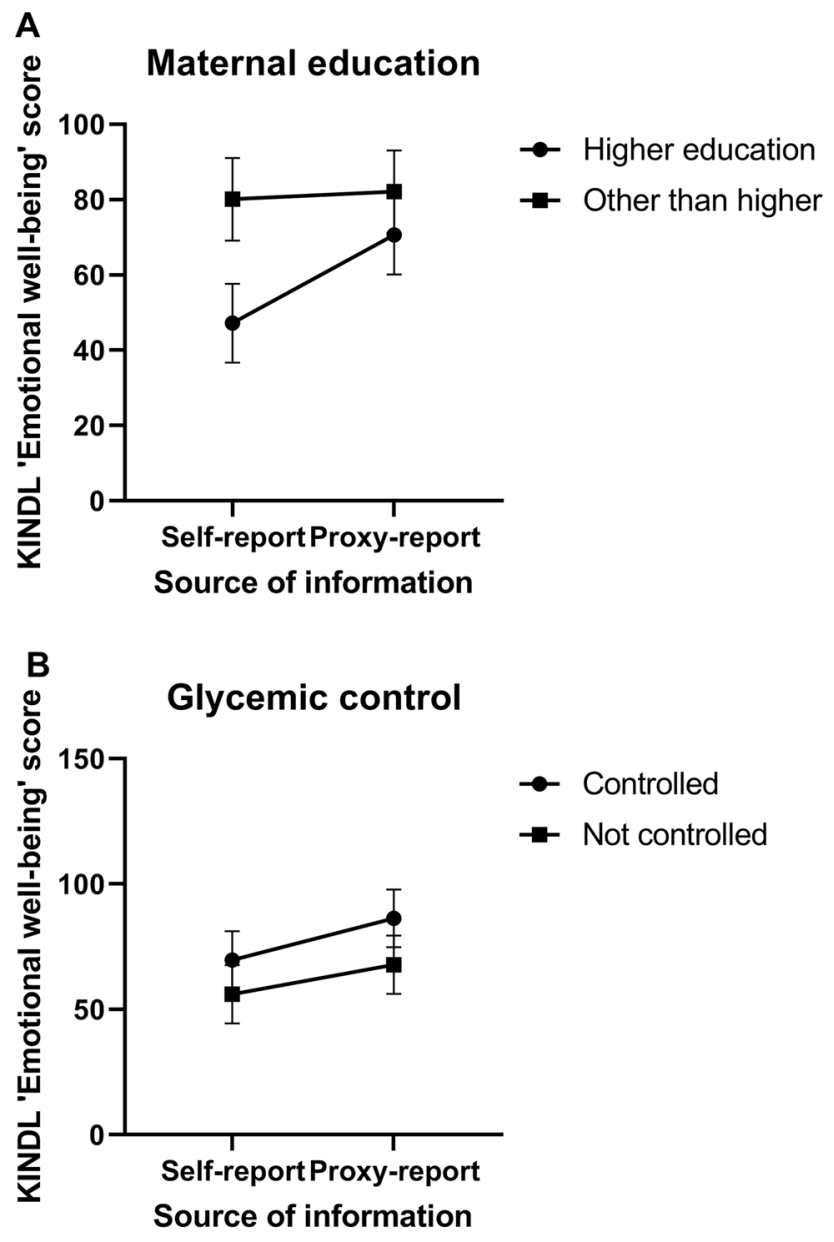

Fig. 3 Relationships between certain modifying factors, self- and proxy-estimates on the KINDL "Emotional well-being" score. Estimated marginal mean values and $95 \%$ confidence intervals

glycemic control was not achieved. Maternal estimates were higher than children's self-reports both in cases of accomplished and non-accomplished glycemic control (Fig. 3B).

\section{Discussion}

This study aimed at comparing self- and maternal proxyreports on different aspects of HRQoL in young children with T1DM.

One finding from this work was that, compared with their children, the mothers provided lower estimates on the KINDL total and the KINDL Disease scales. This finding is generally in accord with a fact that parents of children without chronic illness typically rate their child's HRQoL better than the children themselves, while, in contrast, parents of children with a variety of chronic health conditions, including diabetes, typically rate their child's HRQoL as worse than children themselves [18, 20, 32, 33]. However, across studies, the findings tend to be mixed, with discrepancies commonly reported in both directions [19, 34-37]. Evidently, parents may generally underestimate HRQoL of their ill children due to their concerns about child health, especially considering general health characteristics and disease-specific issues of the quality of life.

An important issue is psychological adjustment in children with T1DM, and the findings were that children with diabetes appear to have a greater incidence of emotional disturbances and psychological distress. Family cohesion, supportive behaviors, and collaborative solving of psychological problems are among the major constituents of care for ill children [5]. In this regard, comparisons between self- and proxy ratings of emotional wellbeing in children with T1DM are of special interest. The findings from this study were that maternal ratings of the HRQoL related to child emotional wellbeing were higher than those in children's self-reports. Previous findings were that concordance among parents and children was greater for physical functioning than for emotional and school functioning [38]. Discrepancies in parents' and healthy children's reports of child emotion regulation were previously reported [39]. Clearly, there is a disconnection between parents' and children' perceptions of emotional state in the young age group. Given that parents are doing much of the diabetes care at this stage, many may undervalue the effect diabetes has on their young child's emotional state. Parents of young children with diabetes may have difficulty in distinguishing diabetes-related feelings, so it may be difficult to accurately assess their child's emotional wellbeing [40]. In the context of clinical care, it is important to consider these discrepancies in HRQoL reports as much as possible, since maternal reporting higher scores can indicate a worrisome lack of correspondence between the mother and the child. If providers note large discrepancies, it may be beneficial to dedicate more time to discussing HRQoL issues with families. However, more research is needed to determine whether HRQoL interventions for families with larger versus smaller discrepancies are impactful [41].

An attempt was made to study possible confounding/modifying effects of several clinical and demographic factors on child-maternal discrepancies in HRQoL estimates. Although some studies were indicative that boys with diabetes tended to report better quality of life [42], no statistically significant effect was found for child sex in this study. Better HRQoL estimates were previously reported in youth with longer diabetes duration and in those from a better socioeconomic background $[19,43,44]$. Other risk factors for unsatisfactory quality of life in diabetic children and adolescents were previously reported to be depression, one-parent family, and diabetes-related family conflict [45]. This study failed to find statistically significant effect of diabetes duration on HRQoL; at least in part, this might be due to relatively 
short and similar diabetes duration in young children who entered this study. Important findings from this study were that child-maternal discrepancies in HRQoL estimates were modified by such factors as maternal marital status, maternal education, insulin regimen, and glycemic control. Generally, higher values of self- and maternal proxy reports were found in those cases where the mothers were married, had education other than higher, when a child received insulin via insulin pump, and when glycemic control was reached. Lower estimates of the child HRQoL in higher-educated mothers and their children might be due to more critical attitudes toward the child state in these families. Insulin pump therapy is the means of intensive treatment and one of the most technologically advanced methods of achieving nearnormal blood glucose levels with numerous benefits ascribed to this regimen, including more flexibility in the timing of patients' meals, exercise, and physical activity [46]. To date, however, few studies have addressed how the adjustment to pump therapy affects HRQoL of children and adolescents. Some findings were indicative that HRQoL was unrelated to regimen prescription in children [47], while the others found insulin pump therapy to be a significant predictor of the HRQoL [48]. Our own findings are the arguments that insulin pump is related to better estimates of HRQoL, both by young children themselves and by their mothers. It is in accord with the reports on the fact that intensive treatment of diabetes does not worsen quality of life and in some cases can even improve it $[12,45]$. Use of the insulin pump does not appear to adversely affect quality of life [12, 47, 49]; instead, it may be associated with improved quality of life, as shown in this present study. In addition, it was found that use of continuous glucose monitoring did not seem to adversely affect quality of life [50].

The relationship between HRQoL and metabolic control, measured by HbA1c, is conflicting [12, 45]. Consistent with our findings, some authors demonstrated that better HRQoL was associated with better glycemic control [51, 52]; poorer HRQoL was associated with higher $\mathrm{HbA1c}$ level and greater depressive symptoms in the pediatric T1DM [49, 53]. Diabetes symptoms are associated with general health-related quality of life, which is partially mediated by diabetes management [54].

Contrasts between children's and maternal reports on the KINDL total estimates in this study were more sharp when the mothers were married, had higher education, and when glycemic control in children was reached. Unlike in this study, some authors found that among adolescents with T1DM, larger discrepancies between self- and parental proxy reports on the generic and diabetes-specific HRQoL modules were associated with poorer glycemic control with higher HbA1c values, in that the youth reported higher scores than their parents [19]. Given adolescents are more likely to have worsening treatment adherence and poor glycemic control [41], it is possible that parental perceptions of adolescent HRQoL may take adherence or glycemic control into consideration, thus rating HRQoL lower when $\mathrm{HbA1c}$ is poor; however, teens' own perceptions of HRQoL may not be related to HbA1c. Differences between parent and youth perspectives on HRQoL that encompass broader family issues, such as family conflict or poor communication in the home, may take place, as both have been associated with HRQoL and HbA1c [55, 56]. By contrast, in young children who may be more compliant with medical and parental recommendations than adolescents and who are more attached to their parents and are closely supervised by them, the reaction of a child and his/her mother to a failure in reaching glycemic control may be similarly frustrating; however, in the event of an achievement of glycemic control, not all maternal expectations about her child progress may be met, while a child may be happy with a success, thus leading to his/her higher estimates of the HRQoL and more discrepancies between self- and proxy reports. Similarly, larger discrepancies between children' and maternal estimates on the total HRQoL scores in the cases when the mothers were married may be due to higher expectations about child health in these families.

Several limitations of this study should be acknowledged. First, the study comprised limited number of 4-to-6-yearold children from the urban setting; thus, the findings from this study cannot be easily expanded across other children categories. Another limitation may come from certain inadequacies between the child and parent KINDL questionnaire forms: except the KINDL Disease scale where both the child and the parental forms each include 6 questions, the remainder scales were based on 2 questions in the child and on 6 questions in the parental form. It is by this that, when using child self-reports, calculation of the KINDL Total score was considered as more reliable estimate [27]. Meanwhile, acceptable-to-good internal consistencies found in all selfand proxy-report subscales argued in favor of meaningfulness of the values obtained, and the score transformation to a 0-100 scale enabled direct comparisons between children's and maternal reports. Another subject of concern may be that although the questions from the KINDL questionnaire were addressing situations related to the last week, the answers of the children might have been somehow influenced by what had happened shortly before testing and by the feelings of the child during the test, i.e., the problem of the test reliability. However, previous studies were indicative of appropriate test-retest stability of the KINDL questionnaires in 4-17-year-old children from different settings using different linguistic versions of the questionnaire, in all the subscales and the total score, even when re-testing has been performed 7 to 21 days after the initial test [57-60]. Even admitting that current life events might somehow have influenced responses, such non-differentiated response bias could 
not invalidate the results of comparisons performed in this study. We must also take into account that numerous exogenous factors remaining beyond the scope of this study might have influenced HRQoL estimates produced by children and their parents. In particular, the study was conducted under the circumstances of the COVID-19 pandemic. Meanwhile, the pandemic did not interrupt the delivery of ambulatory and hospital medical care to children with diagnosed T1DM. The children and their parents who entered this study were local residents, and thus faced principally similar burdens related to the pandemic.

With all the limitations in mind and with full awareness on the fact that further studies in the field are desirable, the findings from this study support prior recommendations to use the child report in conjunction with the parent-proxy report when making determinations on child HRQoL [19, $40,56]$. When child and parent-proxy scores are discrepant, they should be considered individually. Attempts should be made to improve parental understanding of child problems related to his/her disease with special emphasis on child emotional wellbeing with due account to individual family social and demographic characteristics. Clinical assessment and discussion of HRQoL should be considered as a way to comprehensive management of diabetes spanning both physical and mental health.

Supplementary Information The online version contains supplementary material available at https://doi.org/10.1007/s00431-021-04239-0.

Acknowledgements The authors wish to express their gratitude to post-graduate trainees for their help in collecting raw data.

Authors' contributions Irina L. Nikitina: conceptualization and design of the study, review and revision of the manuscript.

Igor A. Kelmanson: conceptualization and design of the study, analysis of data, draft of the initial manuscript.

Availability of data and material Not applicable.

Code availability Not applicable.

\section{Declarations}

Ethics approval All procedures performed in study were in accordance with the ethical standards of the institutional and/or national research committee and with the 1975 Helsinki declaration and its later amendments or comparable ethical standards. The study was approved by the Institutional ethics committee.

Consent to participate Informed consent for the participation in a scientific study was obtained from all mothers involved, and confidentiality was guaranteed.

Consent for publication Not applicable

Competing interests The authors declare no competing interests.

\section{References}

1. Maahs DM, West NA, Lawrence JM, Mayer-Davis EJ (2010) Epidemiology of type 1 diabetes. Endocrinol Metab Clin North Am 39:481-497

2. Centers for Disease Control and Prevention (2011) National diabetes fact sheet: national estimates and general information on diabetes and prediabetes in the United States, 2011. Atlanta, GA: US department of health and human services, centers for disease control and prevention 201:2568-2569

3. Mayer-Davis EJ, Kahkoska AR, Jefferies C, Dabelea D, Balde N, Gong CX, Aschner P, Craig ME (2018) ISPAD Clinical Practice Consensus Guidelines 2018: definition, epidemiology, and classification of diabetes in children and adolescents. Pediatr Diabetes 19(Suppl 27):7-19

4. Monaghan MC, Hilliard ME, Cogen FR, Streisand R (2009) Nighttime caregiving behaviors among parents of young children with type 1 diabetes: associations with illness characteristics and parent functioning. Fam Syst Health 27:28

5. Delamater AM, de Wit M, McDarby V, Malik JA, Hilliard ME, Northam E, Acerini CL (2018) ISPAD Clinical Practice Consensus Guidelines 2018: Psychological care of children and adolescents with type 1 diabetes. Pediatr Diabetes 19:237-249

6. Hilliard ME, Minard CG, Marrero DG, de Wit M, Thompson D, DuBose SN, Verdejo A, Monzavi R, Wadwa RP, Jaser SS, Anderson BJ (2020) Assessing health-related quality of life in children and adolescents with diabetes: development and psychometrics of the Type 1 Diabetes and Life (T1DAL) Measures. J Pediatr Psychol 45:328-339

7. Sinisterra M, Hamburger S, Tully C, Hamburger E, Jaser S, Streisand R (2020) Young children with type 1 diabetes: sleep, health-related quality of life, and continuous glucose monitor use. Diabetes Technol Ther 22:639-642

8. Murillo M, Bel J, Perez J, Corripio R, Carreras G, Herrero X, Mengibar JM, Rodriguez-Arjona D, Ravens-Sieberer U, Raat H, Rajmil L (2017) Impact of monitoring health-related quality of life in clinical practice in children with type 1 diabetes mellitus. Qual Life Res 26:3267-3277

9. Anderson BJ, Laffel LM, Domenger C, Danne T, Phillip M, Mazza C, Hanas R, Waldron S, Beck RW, Calvi-Gries F, Mathieu C (2017) Factors associated with diabetes-specific health-related quality of life in youth with type 1 diabetes: the Global TEENs Study. Diabetes Care 40:1002-1009

10. Cameron FJ (2003) The impact of diabetes on health-related quality of life in children and adolescents. Pediatr Diabetes 4:132-136

11. Clarke SA, Eiser C (2004) The measurement of health-related quality of life (QOL) in paediatric clinical trials: a systematic review. Health Qual Life Outcomes 2:66

12. Wagner VM, Muller-Godeffroy E, von Sengbusch S, Hager S, Thyen U (2005) Age, metabolic control and type of insulin regime influences health-related quality of life in children and adolescents with type 1 diabetes mellitus. Eur J Pediatr 164:491-496

13. Delamater AM (2009) Psychological care of children and adolescents with diabetes. Pediatr Diabetes 10(Suppl 12):175-184

14. Mrkulić E, Mahmutović J, Mizdrak A, Alihodžić A, Lučkin A, Salihović A (2021) Quality of life of children and adolescents with type 1 diabetes mellitus-review. European Journal of Biomedical and Pharmaceutical Sciences 8:65-67

15. Cameron FJ, Smidts D, Hesketh K, Wake M, Northam EA (2003) Early detection of emotional and behavioural problems in children with diabetes: the validity of the Child Health Questionnaire as a screening instrument. Diabet Med 20:646-650

16. Grey M (2009) Coping and psychosocial adjustment in mothers of young children with type 1 diabetes. Child Health Care 38:91-106 
17. Sameroff AJ, Fiese BH (2000) Transactional regulation: the developmental ecology of early intervention. Handbook of early childhood intervention 2:135-159

18. Sattoe JNT, van Staa A, Moll HA (2012) The proxy problem anatomized: child-parent disagreement in health related quality of life reports of chronically ill adolescents. Health Qual Life Outcomes 10:1-13

19. Yi-Frazier JP, Hilliard ME, Fino NF, Naughton MJ, Liese AD, Hockett CW, Hood KK, Pihoker C, Seid M, Lang W, Lawrence JM (2016) Whose quality of life is it anyway? Discrepancies between youth and parent health-related quality of life ratings in type 1 and type 2 diabetes. Qual Life Res 25:1113-1121

20. Eiser C, Varni JW (2013) Health-related quality of life and symptom reporting: similarities and differences between children and their parents. Eur J Pediatr 172:1299-1304

21. Faul F, Erdfelder E, Buchner A, Lang A-G (2009) Statistical power analyses using $\mathrm{G}^{*}$ Power 3.1: Tests for correlation and regression analyses. Behav Res Methods 41:1149-1160

22. Abramson JH (2011) WINPEPI updated: computer programs for epidemiologists, and their teaching potential. Epidemiologic Perspectives \& Innovations 8:1-9

23. DiMeglio LA, Acerini CL, Codner E, Craig ME, Hofer SE, Pillay K, Maahs DM (2018) ISPAD Clinical Practice Consensus Guidelines 2018: glycemic control targets and glucose monitoring for children, adolescents, and young adults with diabetes. Pediatr Diabetes 19(Suppl 27):105-114

24. Ravens-Sieberer U, Bullinger M (1998) Assessing health-related quality of life in chronically ill children with the German KINDL: first psychometric and content analytical results. Qual Life Res 7:399-407

25. Villalonga-Olives E, Kiese-Himmel C, Witte C, Almansa J, Dusilova I, Hacker K, von Steinbuechel N (2015) Self-reported health-related quality of life in kindergarten children: psychometric properties of the Kiddy-KINDL. Public Health 129:889-895

26. Orgilés M, Melero S, Penosa P, Espada JP, Morales A (2019) Parentreported health-related quality of life in Spanish pre-schoolers: psychometric properties of the Kiddy-KINDL-R. Anales de Pediatría (English Edition) 90:263-271

27. Ravens-Sieberer U, Bullinger M (2000) KINDL: Questionnaire for Measuring Health-Related Quality of Life in Children and Adolescents. Revised version. Manual. Hamburg. Retrieved from https://www.kindl.org/english/manual/

28. Ellis PD (2010) The essential guide to effect sizes: statistical power, meta-analysis, and the interpretation of research results. Cambridge University Press

29. Glickman ME, Rao SR, Schultz MR (2014) False discovery rate control is a recommended alternative to Bonferroni-type adjustments in health studies. J Clin Epidemiol 67:850-857

30. Sahai H, Ageel MI (2012) The analysis of variance: fixed, random and mixed models. Springer Science \& Business Media, Boston

31. Jamovi Project (2020) Jamovi (1.0) [Computer software]. Retrieved from https://www.jamovi.org

32. Allan CL, Flett B, Dean HJ (2008) Quality of life in First Nation youth with type 2 diabetes. Matern Child Health J 12(Suppl 1):103-109

33. Nansel TR, Weisberg-Benchell J, Wysocki T, Laffel L, Anderson B, Steering Committee of the Family Management of Diabetes S (2008) Quality of life in children with Type 1 diabetes: a comparison of general and diabetes-specific measures and support for a unitary diabetes quality-of-life construct. Diabet Med 25:1316-1323

34. Hilliard ME, Mann KA, Peugh JL, Hood KK (2013) How poorer quality of life in adolescence predicts subsequent type 1 diabetes management and control. Patient Educ Couns 91:120-125

35. Silva N, Crespo C, Carona C, Bullinger M, Canavarro MC (2015) Why the (dis)agreement? Family context and child-parent perspectives on health-related quality of life and psychological problems in paediatric asthma. Child Care Health Dev 41:112-121

36. Pan R, Egberts MR, Nascimento LC, Rossi LA, Vandermeulen E, Geenen R, Van Loey NE (2015) Health-related quality of life in adolescent survivors of burns: agreement on self-reported and mothers' and fathers' perspectives. Burns 41:1107-1113

37. Murray CB, Holmbeck GN, Ros AM, Flores DM, Mir SA, Varni JW (2015) A longitudinal examination of health-related quality of life in children and adolescents with spina bifida. J Pediatr Psychol 40:419-430

38. Emmanouilidou E, Galli-Tsinopoulou A, Karavatos A, NousiaArvanitakis S (2008) Quality of life of children and adolescents with diabetes of Northern Greek origin. Hippokratia 12:168

39. Hourigan SE, Goodman KL, Southam-Gerow MA (2011) Discrepancies in parents' and children's reports of child emotion regulation. J Exp Child Psychol 110:198-212

40. Hilliard ME, Monaghan M, Cogen FR, Streisand R (2011) Parent stress and child behaviour among young children with type 1 diabetes. Child Care Health Dev 37:224-232

41. de Wit M, Delemarre-van de Waal HA, Bokma JA, Haasnoot K, Houdijk MC, Gemke RJ, Snoek FJ (2008) Monitoring and discussing health-related quality of life in adolescents with type 1 diabetes improve psychosocial well-being: a randomized controlled trial. Diabetes Care 31:1521-1526

42. Anderson BJ, Laffel LM, Domenger C, Danne T, Phillip M, Mazza C, Hanas R, Waldron S, Beck RW, Calvi-Gries F (2017) Factors associated with diabetes-specific health-related quality of life in youth with type 1 diabetes: the Global TEENs Study. Diabetes Care 40:1002-1009

43. Nieuwesteeg A, Pouwer F, van der Kamp R, van Bakel H, Aanstoot H-J, Hartman E (2012) Quality of life of children with type 1 diabetes: a systematic review. Curr Diabetes Rev 8:434-443

44. Hassan K, Loar R, Anderson BJ, Heptulla RA (2006) The role of socioeconomic status, depression, quality of life, and glycemic control in type 1 diabetes mellitus. J Pediatr 149:526-531

45. Laffel LMB, Connell A, Vangsness L, Goebel-Fabbri A, Mansfield A, Anderson BJ (2003) General quality of life in youth with type 1 diabetes: relationship to patient management and diabetes-specific family conflict. Diabetes Care 26:3067-3073

46. Bode BW, Tamborlane WV, Davidson PC (2002) Insulin pump therapy in the 21st century: strategies for successful use in adults, adolescents, and children with diabetes. Postgrad Med 111:69-77

47. Valenzuela JM, Patino AM, McCullough J, Ring C, Sanchez J, Eidson M, Nemery R, Delamater AM (2006) Insulin pump therapy and health-related quality of life in children and adolescents with type 1 diabetes. J Pediatr Psychol 31:650-660

48. Lukacs A, Varga B, Kiss-Toth E, Soos A, Barkai L (2014) Factors influencing the diabetes-specific health-related quality of life in children and adolescents with type 1 diabetes mellitus. J Child Health Care 18:253-260

49. Froisland DH, Graue M, Markestad T, Skrivarhaug T, WentzelLarsen T, Dahl-Jorgensen K (2013) Health-related quality of life among Norwegian children and adolescents with type 1 diabetes on intensive insulin treatment: a population-based study. Acta Paediatr 102:889-895

50. Juvenile Diabetes Research Foundation Continuous Glucose Monitoring Study Group (2010) Quality-of-life measures in children and adults with type 1 diabetes: Juvenile Diabetes Research Foundation Continuous Glucose Monitoring randomized trial. Diabetes Care 33:2175-2177

51. Hesketh KD, Wake MA, Cameron FJ (2004) Health-related quality of life and metabolic control in children with type 1 diabetes: a prospective cohort study. Diabetes Care 27:415-420

52. Hoey H, Aanstoot H-J, Chiarelli F, Daneman D, Danne T, Dorchy H, Fitzgerald M, Garandeau P, Greene S, Holl R (2001) Good 
metabolic control is associated with better quality of life in 2,101 adolescents with type 1 diabetes. Diabetes Care 24:1923-1928

53. Lawrence JM, Yi-Frazier JP, Black MH, Anderson A, Hood K, Imperatore G, Klingensmith GJ, Naughton M, Mayer-Davis EJ, Seid M, SEARCH for Diabetes in Youth Study Group (2012) Demographic and clinical correlates of diabetes-related quality of life among youth with type 1 diabetes. J Pediatr 161:201-207

54. Varni JW, Delamater AM, Hood KK, Driscoll KA, Wong JC, Adi S, Yi-Frazier JP, Grishman EK, Faith MA, Corathers SD (2018) Diabetes management mediating effects between diabetes symptoms and health-related quality of life in adolescents and young adults with type 1 diabetes. Pediatr Diabetes 19:1322-1330

55. Hamman RF, Bell RA, Dabelea D, D’Agostino RB, Dolan L, Imperatore G, Lawrence JM, Linder B, Marcovina SM, Mayer-Davis EJ (2014) The SEARCH for Diabetes in Youth study: rationale, findings, and future directions. Diabetes Care 37:3336-3344

56. Paris CA, Imperatore G, Klingensmith G, Petitti D, Rodriguez B, Anderson AM, Schwartz ID, Standiford DA, Pihoker C (2009) Predictors of insulin regimens and impact on outcomes in youth with type 1 diabetes: the SEARCH for Diabetes in Youth study. $\mathrm{J}$ Pediatr 155:183-189
57. Rajmil L, Serra-Sutton V, Fernandez-Lopez JA, Berra S, Aymerich M, Cieza A, Ferrer M, Bullinger M, Ravens-Sieberer U (2004) The Spanish version of the German health-related quality of life questionnaire for children and adolescents: the Kindl. An Pediatr (Barc) 60:514-521

58. Lin C-Y, Luh W-M, Cheng C-P, Yang A-L, Ma H-I (2014) Evaluating the wording effect and psychometric properties of the KidKINDL. Eur J Psychol Assess 30:100-109

59. Lee PH, Chang LI, Ravens-Sieberer U (2008) Psychometric evaluation of the Taiwanese version of the Kiddo-kINDL generic children's health-related quality of life instrument. Qual Life Res 17:603-611

60. Ravens-Sieberer U, Bullinger M (2001) The revised KINDL-R: final results on reliability, validity and responsiveness of a modular HRQOL instrument for children and adolescents. Qual Life Res 10:199

Publisher's Note Springer Nature remains neutral with regard to jurisdictional claims in published maps and institutional affiliations. 